\title{
The Source and Consequences of Stop Orders: A Conjecture
}

\author{
ADRIAN E. TSCHOEGL \\ Graduate School of Business Administration, The University of Michigan, Ann Arbor, Ml, USA
}

\section{INTRODUCTION}

In United State's stockmarkets investors may place a variety of orders with brokers. One common type of instruction is the stop ('stop loss') order, where the investor instructs the broker to sell (buy) if the market price goes below (above) some predetermined price. Such orders are not implicit options. They are contingent instructions, not contingent claims. The broker is simply an agent for the investor, monitoring and acting on prices on the investor's behalf. The broker assumes no risk.

The common reasons for issuing stop orders are (Eiteman et al., 1966):

(1) To limit a loss on the long or short side of the market;

(2) To protect a gain on the long or short side of the market.

However, such orders are meaningless or even not rational under the now common random walk model for stock price behavior.

One can make an argument for stop sell orders for tax reasons. That is, investors could instruct brokers to sell if the share price falls below the price at which the investor bought. In this way the investor could perhaps reduce monitoring costs. However, this still leaves buy orders unexplained.

In this note we conjecture that stop orders are automatic devices which investors use to enable them to react to price changes prior to receiving the information that caused the price to change. Subsequent sections discuss models of price behavior, approaches to setting stop prices and the consequences of myopic use of such orders.

\section{MODELS OF STOCK PRICE BEHAVIOR}

A discrete time geometric random walk model for stock prices is:

$$
\ln \tilde{P}_{i+1}=\ln P_{i}+\tilde{\varepsilon}_{i+1}
$$

where $P_{i}$ is the asset price at the end of the $i$ th period, $\tilde{\varepsilon}_{i+1}$ is the stochastic return and the sequence $\left\{\varepsilon_{i}\right\}$ is identically and independently distributed as $N\left(\mu, \sigma^{2}\right)$. Tildes indicate random variable as of the end of period $i$. This model implies that for every positive integer $k, \rho(k)$, the autocorrelation of $\tilde{s}_{i}$ at a lag of $k$ periods, is zero.

Under this model, how the stock price reached the stop price says nothing about the price's future behavior. There is no reason to stop a loss or to protect a gain as the next change, net of the expected return $\mu$, is as likely to be gain as a loss, or vice versa. If placing a stop order rather than buying 'at market' involves any extra costs at all, the expected value of the stop order is negative. However, this geometric random walk model may provide a poor description of stock price behavior, at least in the short run.

A number of authors, reviewed in Nawrocki (1984), have suggested disequilibrium models in which there is a finite speed of price adjustment to new information because of transaction costs, taxes, short sales restrictions, costs of acquiring and evaluating information and noisy information systems. Hasbrouck and Ho (1986) find evidence for lagged adjustment in intra-day data, where the rate of adjustment is positively related to the market value of the stock. Copeland (1976) has suggested a model in which new information disseminates to investors sequentially. In the Copeland model the proportion of informed and uniformed traders changes as the information disseminates through the market. Because adjustment towards equilibrium spans periods, observed returns may be autocorrelated, and exhibit temporary substantial positive or negative trends. Then the problem facing investors is how to avoid being at a disadvantage in their transactions when, because of their distance from the market, they can expect to be among the last to receive new information.

\section{STOP ORDERS AS CONTROL LIMITS}

We suggest that stop orders provide a way for investors to react to shifts in the underlying process, even though they may not know why the shift has occurred. As such, they could speed the rate of adjustment of prices to the new equilibrium. Stop orders may represent a means of 
implementing control limit procedures in a way that shifts the monitoring function to brokers for whom the marginal costs are lower than for investors. There are a number of ways of determining such intervention points.

Brown (1971), building on Wald's (1947) seminal work on sequential analysis, has developed a computationally feasible model which detects shifts in the underlying process as quickly as possible after they happen. He develops a parabolic mask centered on the most recent observation and based on the null hypothesis of no change in $\mu$. The price series crossing the mask provides the decision rule for rejecting the null hypothesis of no change in the trend.

Assuming Eqn.(1), and with no loss of generality that $\mu=0$, the formula for the mask over the interval $k(k=1, \ldots, n)$ is:

$$
P_{k}=P_{0} \exp \pm(\sigma \sqrt{ } k) \sqrt{ }\{2 \ln [(1-\beta) / \alpha]\}
$$

where $\alpha$ is the investor's choice of the risk of incorrectly accepting that the expected return has changed when it has not and $\beta$ that of not detecting a change which has occurred. That is, the mask is the product of the standard deviation of the process times the square root of the number of periods into the future, adjusted to take into account the probabilities of Type I and Type II errors.

This formula is appropriate if we model the affect of lagged adjustment as a change in trend (Hasbrouck and Ho, 1986; Taylor, 1982). If we model it as temporary positive serial correlation (Copeland, 1976; Tschoegl, 1986), we would require a different formulation. In either case, the observed price path will have a higher probability of crossing the mask than under the null hypothesis of no change in trend or no positive autocorrelation.

Brown's formula is simply one parametically based approach to the problem. Nawrocki (1984) introduces an adaptive feature which narrows or widens the parabola depending on the first order autocorrelation coefficient calculated for the previous period, and applies the mask to the cumulative sum of forecast errors from a moving average model of stock prices.

So-called charists or technical anaysts use other procedures such as the comparison, using daily data, of the nine- and twenty-six-day moving average. They also use more complicated and subjective rules involving volume, high and low prices, and other data. The common ground of all these approaches is an attempt to extract a signal of a change in trend from noisy data using sequential observations. The use of these approaches, combined with the use of round numbers as 'focal' points (Schelling, 1960; Durand, 1961; Neiderhoffer, 1965), may be the source of the 'psychological barriers', frequently referred to by stockmarket practitioners, whose crossing by the price will trigger extensive buy or sell orders. The use of these devices to determine when an adjustment had run its course in order to reverse a position entered into in response to a previous signal also provides an explanation for the common stock market expression, "widespread profit taking'.

\section{PROBLEMS AND CONSEQUENCES}

These trend change detection devices suffer from empirical and conceptual problems. The empirical problem is the assumption that trends are long-lived, that is that they may extend for many days. The evidence from the event studies literature indicates that for most of the information events examined all of the price adjustment occurs within a day or so. In addition, filter rule tests and other tests of mechanical rules on daily data have consistently failed to beat buy-andhold strategies. Even Nawrocki's (1984) adaptive mask did not prove more profitable than buy-and-hold when used on monthly data.

This may simply mean that the rules are more appropriate for intra-day data (though one would have to adjust for the data's non-normality). Again, Hasbrouck and Ho's (1986) results for transactions data indicated that for stocks with large market values, adjustment of market prices to 'equilibrium prices was approximately complete within the day. That is, trends, dissipated within a day. While there is almost no information in the academic literature about the number and duration of stop orders, by exchange rules stop orders expire at the end of the trading day unless revoked or executed earlier. The more serious conceptual problem with these automatic devices is the implicit assumption that using them does not affect prices.

While perhaps no one trader's use of these devices will affect prices substantially, many traders' use could. Harris and Gurel (1986) find evidence that the sum of many small transactions unaccompanied by information effects can cause temporary price changes. French and Roll (1985) investigate the hypothesis that trading is self-generating and find that on average, between $4 \%$ and $12 \%$ of daily stock return variance is caused by mispricing. Investor's reaction to exogenously induced trades should not introduce additional variance into price changes. However, this requires that automatic decisions rules take into account the possibility of overreactions. If they do not, this myopia could contribute to the often observed higher volatility of prices during hours when exchanges are open than when they are closed.

\section{Acknowledgement}

The author would like to thank Clifford A. Ball for his helpful comments on a previous draft. 


\section{REFERENCES}

R. G. Brown (1971). Detection of turning points in a time series. Decision Sciences 2, 383-403.

T. E. Copeland, (1976). A model of asset trading under the assumption of sequential information arrival. Journal of Finance 31 (4), 1149-68.

J. Durand (1961). L'attraction des nombres rondes et ses conséquences economiques. Revue Française de Sociologie 11. 131-51

W. J. Eiteman, C. A. Dice and D. K. Eiteman (1966). The Stock Market, 4th edn, New York: McGraw-Hill.

$K$. French and R. Roll (1985). Stock return variances: the arrival of information and the reaction of traders. UCLA Graduate School of Management, Working Paper 3-84, July.

L. Harris and E. Gurel (1986). Price and volume effects associated with changes in the S\&P 500 list: new evidence for the existence of price pressures. Journal of Finance 41 (4), 815-29.
J. Hasbrouck and T. S. Y. Ho (1986), Intraday stock returns: empirical evidence of lagged adjustments. New York University, Solomon Brothers Center for the Study of Financial Institutions, Working Paper 376, May.

D. Nawrocki (1984). Adaptive trading rules and dynamic market disequilibrium. Applied Economics 16, 1-14.

V. Niederhoffer (1965). Clustering of stock prices. Operations Research 13, 258-65.

T. C. Schelling (1960). The Strategy of Conflict, Cambridge, MA: Harvard University Press.

S. J. Taylor (1982). Tests of the random walk hypothesis against a price trend hypothesis. Journal of Financial and Quantitative Analysis 17, (1), 37-61.

A. E. Tschoegl (1986). The effect of market micro-structure on observed prices: the case of the London gold market. University of Michigan, unpublished paper.

A. Wald (1947). Sequential Analysis, New York: John Wiley. 